\title{
Plädoyer für NIDCAP in Deutschland
}

PFLEGEKONZEPT FÜR FRÜHGEBORENE Intensivmedizinische Maßnahmen haben einen enormen Einfluss auf das Verhalten und die Entwicklung Frühgeborener. Um ihnen trotz der belastenden Eingriffe eine optimale Entwicklung zu ermöglichen, benötigen sie eine besondere, individuelle Betreuung. Das NIDCAP-Konzept fördert gezielt die Hirnentwicklung und stärkt die Bindung des Frühgeborenen zu seiner Familie.

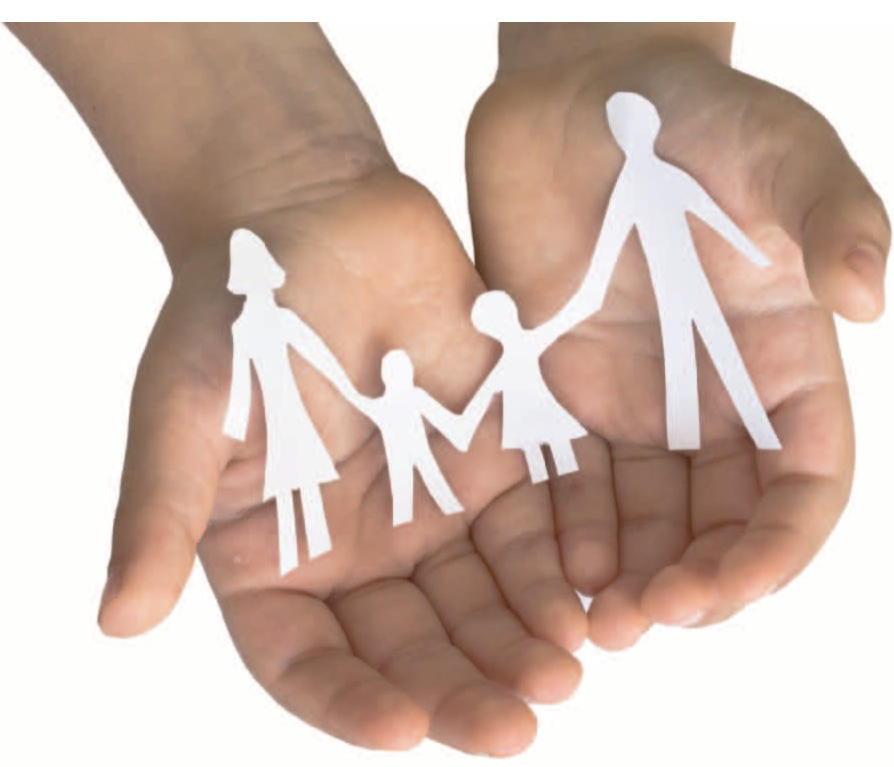

Obwohl das Konzept NIDCAP bereits seit über 28 Jahren in den USA angewendet wird und anhand von Studien überprüft wurde, ist der Begriff für viele neu oder mit falschen Inhalten gefüllt.

Die Tatsache, dass das von Heideliese Als entwickelte Konzept zur Verbesserung des kognitiven und körperlichen Outcomes Frühgeborener bereits weltweit implementiert ist und gute Erfolge aufzeigt, lässt die Frage aufkommen, warum Deutschland von der Zukunftsvision NIDCAP bisher noch relativ unberührt ist.

Trotz verbesserter Überlebenschancen sehr kleiner Frühgeborener seit der Entstehung der Neonatologien in den Sechzigern ist das Outcome häufig weiterhin geprägt von Seh- und Hörschäden, mentaler Retardierung, Sprachschwierigkeiten, Schulproblemen, Verhaltensstörungen und Störungen im Sozialverhalten [1, 2]. Durch die Umsetzung des NIDCAP-Konzepts erleiden Frühgeborene weniger körperliche und mentale Entwicklungsstörungen $[1,3]$ und profitieren von einer geringeren Beatmungsdauer, weniger sauerstoffpflichtigen Tagen, einer rascheren oralen Ernährung und Entwöhnung von der Ernährungssonde sowie einem insgesamt kürzeren Krankenhausaufenthalt. Gewicht, Länge und Kopfumfangsdaten sind bei der Entlassung aus dem Krankenhaus angestiegen. Ebenso erkranken diese Kinder vergleichsweise we- niger an IVH (Ventrikelblutung), BPD (Bronchopulmonale Dysplasie), NEC (nekrotisierende Enterokolitis) und ROP (Retinopathia praematurorum). Weitere Vorteile sind die Jobzufriedenheit des medizinischen Personals und die Senkung des Stresspegels der Eltern. Geringere Behandlungskosten und ein verbessertes Outcome legen die Annahme nahe, dass dieses Konzept das zurzeit beste zur Versorgung frühgeborener Kinder ist [1, 4, 5].

Was genau hat man sich nun darunter vorzustellen? Wie wird NIDCAP eingeführt und welche Veränderungen bringt es mit sich? Und ist dieses Konzept in Deutschland überhaupt durchführbar?

\section{Ausbildung}

NIDCAP (Newborn Individualized Developmental Care and Assessment Program) ist ein umfassendes Konzept für die Betreuung von Frühgeborenen im Umfeld einer Intensivstation. Das Gehirn des Fetus macht ein rapides Wachstum zwischen der 24. und 40. Schwangerschaftswoche (SSW) durch. Nervenzellen und Gliazellen vermehren sich, die Myelinisierung beginnt, es entwickeln sich Dentriten und Synapsen, 70\% der kortikalen Neurone unterliegen der Apoptose, die graue Substanz nimmt um bis zu 400\% zu, Gyri und Sulci bilden sich aus. Diese Entwicklung wird durch „zu erwartende Reize“ der plazentaren Umgebung unterstützt. Erleidet ein Kind zu diesem Zeitpunkt eine Frühgeburt, wird es mit unphysiologischen Reizen konfrontiert und die physiologische Entwicklung des zentralen Nervensystems kann daran Schaden nehmen. Die verpasste Hirnentwicklung durch nicht erlebte zu erwartende Reize kann nicht nachgeholt werden [6]. NIDCAP ist ein Programm, das den Reifungsprozess des zentralen Nervensystems fördert, indem es die Umgebungsreize der Umwelt des Kindes individuell gestaltet - auf das jeweilige Entwicklungsstadium und dessen Bedürfnisse sowie die aktuelle körperliche Stabilität angepasst. Dadurch wird eine Veränderung in Struktur und Funktion des Gehirns bewirkt. Die Folgen sind eine bessere motorische Organisation, ein höheres Maß an Aufmerksamkeit und ein ausgeglicheneres Verhalten [6, 7].

Das Programm wurde von Heideliese Als und ihren Kollegen entwickelt und 1982 erstmals in einer Klinik in Boston, USA, eingeführt. Eine Aufgabe der Pflege ist die direkte Beobachtung des Frühgeborenen in seiner Umwelt und in den Gegebenheiten der Intensivpflege sowie die Einschätzung mittels spezifiziertem APIB Scoring System. Mit dessen Hilfe kann das Verhalten des Kindes interpretiert und daraufhin eine entwicklungsfördernde Betreuung geplant werden. 
Das NIDCAP-Modell beschreibt die Erschaffung eines entwicklungsfördernden Milieus von räumlichen Strukturen bis zur pflegerischen Betreuung. Die Umsetzung erfordert fortlaufende Schulung und gute Zusammenarbeit des gesamten Teams - von der Pflegenden bis zur Verwaltungsleitung [8].

Die Ausbildung kann in mehreren Levels absolviert werden, wobei nur das erste notwendig ist, um das Konzept zu implementieren.

Qualifiziert für die Level-1-Ausbildung sind Personen, die zum Team einer Neugeborenen-Intensivstation gehören oder interdisziplinär in Kontakt mit diesem stehen. Die Implementierung dauert in der Regel fünf Jahre, die Eröffnung eines Ausbildungszentrums bis zu zehn Jahre. Auf einer Station beinhaltet sie die Ausbildung von mindestens zwei Level-1-Spezialisten, deren Aufgabe es ist, den fortlaufenden Prozess der NIDCAP-Implementierung zu unterstützen, zu evaluieren und mit regelmäßigen Schulungen des Personals voranzutreiben.

Die Station sollte ein interdisziplinäres Leitungsteam zusammenstellen, das den Prozess der Einführung gemeinsam mit den NIDCAP-Spezialisten unterstützt [9].

\section{Basis NIDCAP-Ausbildung oder NIDCAP Level 1}

Die Basis NIDCAP-Ausbildung ist international in 16 Trainingcentern möglich, fünf davon sind in Europa: in Frankreich, Belgien, England, den Niederlanden und Schweden.

Vor Beginn der Ausbildung müssen ca. 70 Fachartikel gelesen werden, um ein gewisses Basiswissen zu erlangen. Viele der Artikel stammen aus der amerikanischen Fachzeitschrift „Early Human Development“, einige davon sowie weitere Basisliteratur sind im Literaturverzeichnis aufgeführt (z.B. $[1,18]$ und $[20]$ ).

Der erste Ausbildungsschritt findet als dreitägiger Block statt: Basisunterlagen werden ausgehändigt, ein Überblick über die Hilfsmittel zur Verhaltensbeobachtung wird gegeben und Organisatorisches besprochen.

Der Auszubildende übt sich nach dem Einführungsblock in der Verhaltensbeobachtung und soll im Laufe der Ausbildung 20 Beobachtungen machen: vom kritisch kranken bis hin zum gesunden Kind.

Nach dem selbstständigen Teil findet wieder ein dreitägiger Block statt, der eine Begleitung während der Arbeit und ein anschließendes Feedback beinhaltet. Im Anschluss folgt das Praktikum für Fortgeschrittene, wobei der Auszubildende ein frühgeborenes Kind mit sehr niedrigem Geburtsgewicht vom Tag seiner Geburt bis zu seiner Entlassung beobachtet und einen wöchentlichen Bericht schreibt.

Der abschließende dreitägige Block beinhaltet wieder eine Beobachtung während der Arbeit und ein anschließendes Feedback [9]. Der nun ausgebildete NIDCAP-Spezialist ist laut Browne kein einzelner Arbeiter mehr, er sollte vielmehr gleichermaßen als Leiter und als Mitglied des Entwicklungsteams fungieren. Er sollte entscheiden, wie das Pflegeteam mit Informationen unterstützt werden kann, wie Interventionsstrategien an die Eltern und versorgende Schwester weitergegeben werden können und wie diese richtig angebracht werden. Die Basisausbildung nimmt den Erfahrungen nach etwa ein bis zwei Jahre in Anspruch [10]. Stationen, die das NIDCAP-Konzept bereits eingeführt haben, können für eine gute Umsetzung der Prinzipien die NIDCAP Nursery Zertifizierung erlangen. Beurteilt werden die räumlich-strukturelle Umsetzung und die Umsetzung in der Pflege, die Philosophie und Einführung in der Pflege des Kindes, in der Familie und beim Personal. Bisher wurden von der NFI (NIDCAP Federation international) zwei Stationen zertifiziert [8].

\section{Spezielle NIDCAP-Ausbildung oder NIDCAP Level 2}

Zielgruppe sind ausgebildete NIDCAP-Level-1-Spezialisten und Personen, die APIB als Instrument für wissenschaftliche Zwecke nutzen wollen. In dieser Ausbildung werden spezielle Kenntnisse in der neurologischen Verhaltensbeurteilung vermittelt.

APIB (Assessment of Preterm Infants Behavior) ist ein Hilfsmittel zur neurologischen Verhaltensbeobachtung bei Früh- und Neugeborenen zur Planung der Pflege und zur Förderung nach dem NIDCAP-Prinzip, entwickelt von Heideliese Als und ihren Kollegen.

APIB integriert die Feinheiten der frühgeborenen Kinder, in ihren Bemühungen nach Selbstregulation und ihren Kapazitäten mit Störungen umzugehen. Zu beobachtende Bereiche sind die fünf Subsysteme

- Autonomie (Hautkolorit, Darmmotorik, Herz- und Atemfrequenz),

- Motorik (Muskeltonus, Körperhaltung, Mimik),

- Bewussteinsstadien (Schlaf-Wach-Rhythmus, Schreien),

- Aufmerksamkeit (Interaktion mit dem Umfeld),

- Selbstregulation (Hand-Mund-Koordination, Greifen).

Selbstregulation bezeichnet die Fähigkeit eines Früh- und Neugeborenen, die Funktionen der fünf Subsysteme stabil aufrechtzuerhalten. Dadurch ist das Kind in der Lage, sich in Stresssituationen zu beruhigen und sich somit selbst zu trösten [3]. Vor der Ausbildung wird eine NIDCAP-Zertifizierung empfohlen.

\section{Umsetzung}

Die Vorgaben der NFI sind relativ allgemein gehalten und werden mithilfe des NIDCAP-Ausbilders individuell für die betreffende Klinik umgesetzt. Die offiziellen Vorgaben beschreiben im Allgemeinen die Reduktion von Licht und Geräuschen, die individuelle Einschätzung jedes Kindes und das Ausrichten der Betreuung danach sowie die grundsätzliche Schmerzreduktion durch Unterstützen der Selbstregulation des Kindes, die bestmögliche Elternintegration in die Pflege und eine starke Förderung der Familienzusammenführung. Nachfolgender Abschnitt ist eine Informationssammlung aus Studienergebnissen, veröffentlichten Fachartikeln sowie den Pflegeleitlinien der NFI.

\section{Die Familie}

Der Raum um das Kind herum sollte ausreichend Möglichkeiten zum Känguruhen, Ausruhen und zur Übernachtung für die Eltern bieten. Die Familie soll dazu ermutigt werden, sich intensiv in die Betreuung des Kindes einzubringen. Das kann die Versorgung ihres Kindes, die Teilhabe an Visiten, Übergaben und Besuchen durch das Geschwisterkind bedeuten.

Für mehr Privatsphäre sollte der Platz des Kindes vom Betrieb der Station abgeschirmt sein. 


\section{Das Kind}

Physiologische Positionen sollten in jeder Lage unterstützt werden. Beim Bewegen oder Umlagern des Kindes müssen die Arme und Beine immer in leichter Flexion unterstützt werden $\rightarrow$ Abb. 1, 2). Die beiden Hände des Versorgers sollten jeweils die Rückund Vorderseite des Kindes umfassen, während Kopf und Extremitäten in der Mittellinie des Körpers gehalten werden. Es sollte nur in dieser Position bewegt und hochgehoben werden, da sie ihm die größte Unterstützung zur Selbstregulation bietet.

Alle Bewegungen werden langsam durchgeführt. Wird das Kind unruhig, nachdem die Hände den Körper verlassen haben, erfährt es erneute Unterstützung, bis es zur Ruhe gekommen ist.

Die Versorgung des Kindes sollte möglichst nach dem Konzept der Bezugspflege geschehen, um die Anzahl der pflegenden Personen gering zu halten und um ihm Sicherheit durch Vertrautheit zu geben. Das Herangehen an das Kind richtet sich nach seinem Schlafund Wachrhythmus. Wenn möglich, sollten sich alle Therapie- und Diagnoseverfahren sowie die Versorgung danach richten und in den Wachphasen des Kindes stattfinden.

Über die Frequenz und den Zeitpunkt von Routinehandlungen wird individuell und unter Berücksichtigung der Energiereserven entschieden. Nach allen Manipulationen wird das Kind so lange gehalten, bis es zur Ruhe gekommen ist. Auf Weinen oder Unwohlsein des Kindes wird sofort reagiert.

Ein wichtiges Kontingent in der entwicklungsfördernden Pflege ist das Management von Schmerzen und Stressreizen, um eine Schädigung des Hirns zu vermeiden. Wie sich in Studien gezeigt hat, ist das frühgeborene Kind zwar in der Lage, nozizeptive Stimuli zu empfinden, allerdings reagiert die Ausschüttung endogener Opiate zur Schmerzhemmung verzögert und die Reizschwelle für Schmerzempfindung ist deutlich niedriger, was die Frühgeborenen sensibler für Schmerzen macht [11].

Studien von Corff et al. 1995 sowie Bellieni et al. 2001 zeigten, dass die emotionale und sensitive Unterstützung - wie das „facilitated tucking“ des Kindes - neben dem nicht nutritiven Saugen die effektivste Methode ist, Schmerz zu lindern. Hierbei liegt das Kind in Seiten- oder Bauchlage, Arme und Beine werden in leichter Beugehaltung durch die Hände der Pflegeperson unterstützt. Eine Verstärkung des analgetischen Effekts bietet die Kombination mit nicht nutritivem Saugen an einem Beruhigungssauger mit Glukose $10 \%$ - ein einfaches und wirksames analgetisches Hilfsmittel und auf Intensivstationen durchaus anwendbar $[3,12,13]$.

Ein wichtiges Instrument zur Beurteilung des Verhaltens ist die APIB-Skala, in der Stresssignale anhand der oben beschriebenen fünf Subsysteme unterteilt werden. Autonome Stresssignale sind z. B. Würgen, Spucken, Niesen und Gähnen sowie Apnoen oder Veränderungen des Hautkolorits. Motorische Stresssignale sind z.B. Hypotonie an Stamm und Extremitäten, Hyperextension der Beine, Salutieren und die Arme schützend nach oben halten ( $\rightarrow$ Abb. 3, 4).

\section{Die Station}

Laut Als ist der Schritt weg vom protokollgeleiteten hin zum individuellen, beziehungsorientierten Betreuungskonzept die größte Veränderung. Die Station muss anfangen, sich nicht mehr als räumliche Pflegeeinrichtung zu begreifen, sondern als solche, die
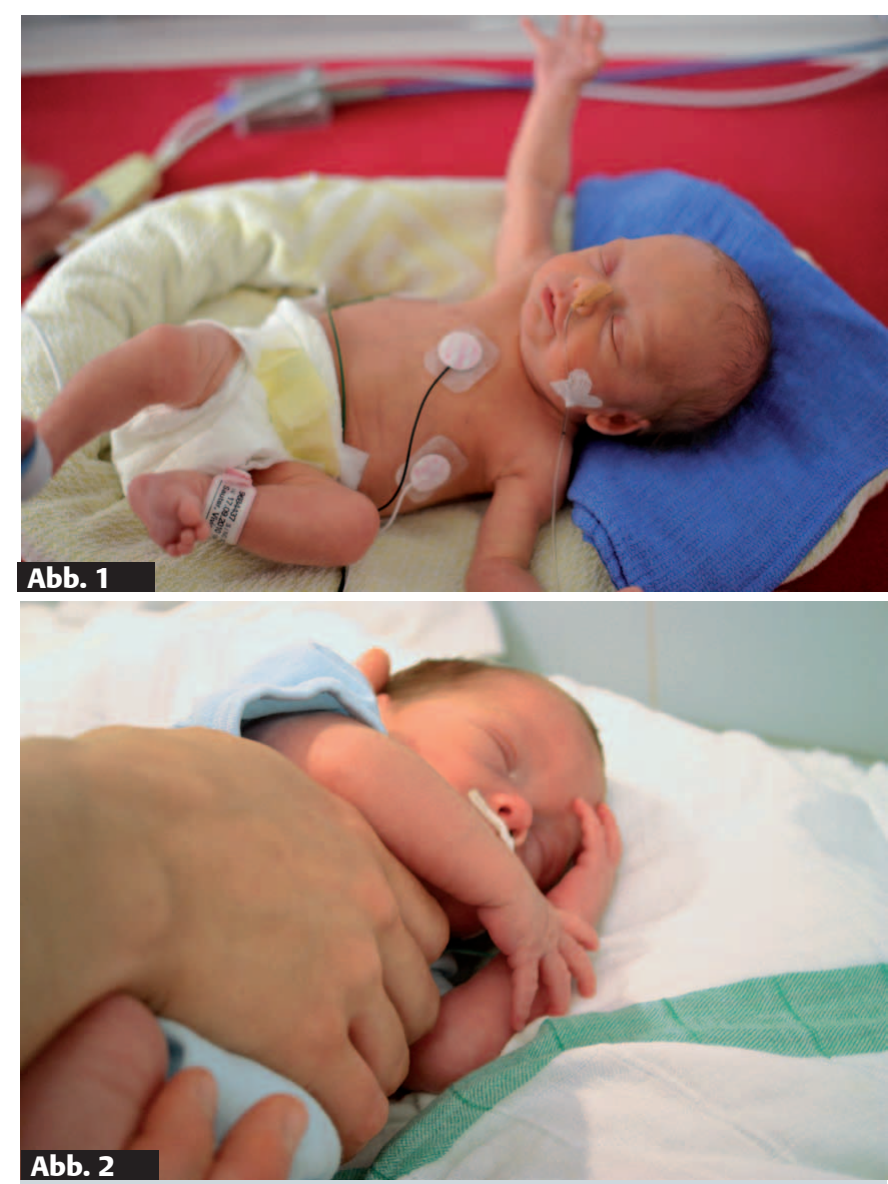

Abb. 1 Hier findet keine Unterstützung der Selbstregulation statt, die Arme liegen weit abgespreizt von der Mittellinie des Körpers.

Abb. 2 Arme und Beine werden in Flexion zur Mittellinie unterstützt.

die Entwicklung von Kind und Familie fördert [14]. Das Team leitet die Eltern an, auf das Verhalten ihres Kindes einzugehen und sich während Interventionen sicher zu fühlen.

Das Licht der Intensivstation hat direkten Einfluss auf die physiologische Stabilität und die Funktion des zentralen Nervensystems des frühgeborenen Kindes. Das Committee for Recommended Environmental Standards in the NICU und die American Academy of Pediatrics empfehlen, dass die Lichtintensität im Durchschnitt nicht mehr als 600 Lux betragen sollte, zur adäquaten visuellen Beobachtung sollte sie nicht höher als 1.000 Lux liegen. Die für die Hirnentwicklung so wichtigen REM-Schlafphasen kommen bei hohen Lichtverhältnissen nur in niedrigerer Frequenz vor [6, 15], deshalb ist es besonders wichtig, Dunkelheit während des Schlafes sicherzustellen.

Auf das kindliche Auge darf nur indirektes Licht fallen. Falls eine Beurteilung bei hohen Lichtverhältnissen nötig ist, müssen die Augen des Kindes unter allen Umständen abgeschirmt werden. Korones fand heraus, dass ein Früh-/Neugeborenes auf der Intensivstation bis zu 134-mal in seinem Schlaf unterbrochen wird - neben der 

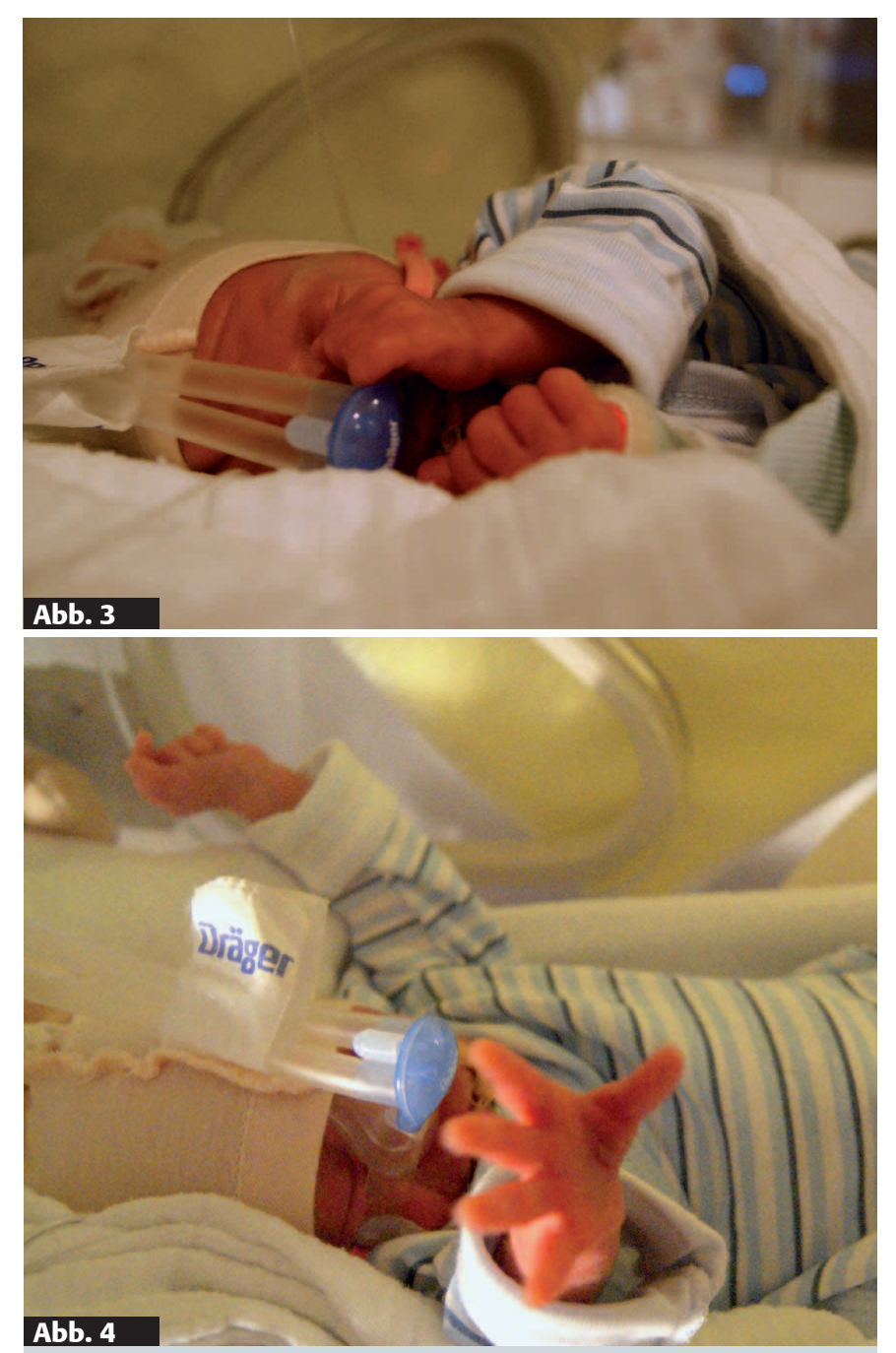

Abb. 3 Selbstregulatorisches Verhalten: die Arme werden selbstständig zur Mittellinie und die Hände zum Gesicht geführt.

Abb. 4 Motorisches Stresszeichen nach APIB: Die Arme werden schützend nach oben gehalten.

Lichtreduktion ist also die Bündelung der Maßnahmen auf einen Zeitpunkt, an dem das Kind wach ist, vorzunehmen [4].

Frühgeborene Kinder sind durch ihre Unreife besonders verletzlich durch Lärm. Die arterielle Sauerstoffsättigung nimmt bei hohen Umgebungsgeräuschen signifikant ab. Hohe Geräuschpegel oder -spitzen machen das frühgeborene Kind zu einem Risikopatienten für lärminduzierte Hörschäden $[4,12,16,17]$.

\section{NIDCAP in Europa}

Das NIDCAP-Konzept ist in Europa in etwa 27 NICUs implementiert und wird in 26 NICUs eingeführt ( $\rightarrow$ Abb. 5). Neben den aufgeführten Ländern verfügt auch Schweden über ausgebildete Units, zu denen es leider keine konkreten Angaben gab.

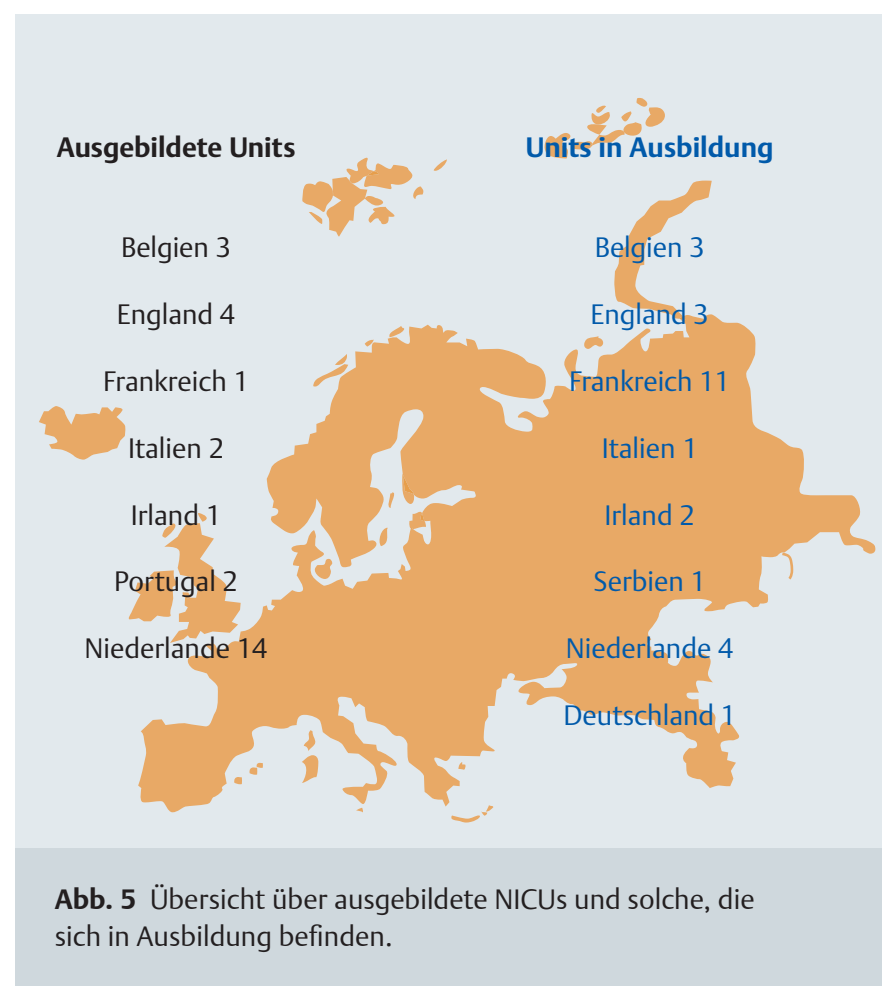

\section{Kosten der Umstellung}

Die Kosten für die Ausbildung der Mitarbeiter belaufen sich laut Westrup auf etwa 5.000 US-Dollar pro Person. Hinzu kommen die Reise- und Unterbringungskosten des Ausbilders und der Ausfall von ein bis zwei Vollzeitarbeitskräften, die für die Ein- und Weiterführung des Programms zuständig sind. Eventuell müssen Umbaumaßnahmen oder Anschaffungen mit eingeplant werden [18]. Das Einsparpotential durch die Umstellung beläuft sich laut einer Studie von Als im Durchschnitt auf 90.000 US-Dollar pro Kind. Laut einer Studie von Fleisher et al. betrug die Ersparnis jeweils 128.670 US-Dollar. Zudem sind die Pflegekosten in Personal- und Liegedauerkriterien geringer, ebenso wie der Pflegeaufwand pro Kind [19].

\section{Vorreiter Universitätsklinikum Heidelberg}

Das Universitätsklinikum Heidelberg entwickelte 2005 ein Konzept auf den Grundlagen des NIDCAP: die Entwicklungsfördernde Familienzentrierte Individuelle Betreuung (EFIB) wurde auf die Struktur deutscher Kliniken angewandt. Die EFIB ist ebenfalls ein Konzept zur Umgebungsgestaltung einer Neugeborenenintensivstation und vermittelt neben den Grundsätzen des NIDCAP die auf Erfahrungen beruhenden Grundsätze zur Umsetzung im Stationsalltag.

In der angebotenen Qualifizierungsmaßnahme werden intra- und extrauterine Entwicklung, Stresserkennung, Förderung der Entwicklung und die Auswirkungen auf den Stationsalltag vermittelt. Während der beiden Ausbildungsblöcke erhalten die Teilnehmer ins Deutsche übersetztes Material der NIDCAP-Literatur und Arbeitsmaterial zur Umsetzung auf der eigenen Station. Die Qualifizierung kostet 600 Euro pro Person [20]. Zeitgleich zur 


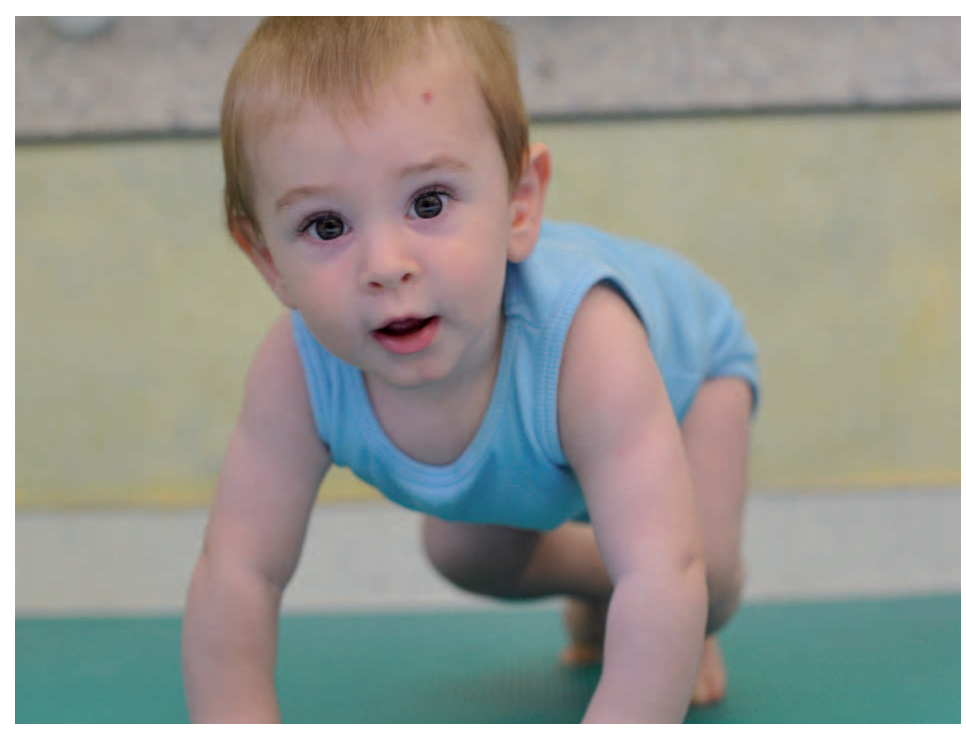

Wir wollen nicht nur, dass die Kinder die Schicht, die Nacht,

die Zeit auf der Intensivstation und im Krankenhaus über-

stehen, sondern wir wollen natürlich auch, dass sich die

Frühgeborenen körperlich und geistig gut weiterentwickeln

und ein gesundes Leben führen können.

Professor Dr. Otwin Linderkamp, Universität Heidelberg

Entwicklung des EFIB-Konzepts startete die Implementierung des NIDCAP durch die Ausbildung zweier Mitarbeiter [21].

\section{Die Compliance der Mitarbeiter}

Als und Gilkerson sagen, weil NIDCAP ein beziehungs- und systemorientiertes, prozessgesteuertes und nicht nur auf Prozeduren basierendes Konzept ist, können Schwierigkeiten entstehen, will man das Konzept in einer Neonatologie einführen, die auf medizinische Protokolle und Pflegeroutinen fokussiert ist [22]. Die bisherige skeptische Einstellung könnte auf dem Denken basieren, man benötige nicht noch ein weiteres Konzept, da man schon Konzepte wie Basale Stimulation oder Kinästhetik infant handling anwendet. Während diese sich zwar mit Wahrnehmungsförderung, Kommunikation durch Berührung und ganzheitlicher Betrachtung des Menschen befassen, nehmen sie jedoch keine neurologische Einschätzung der Situation des Kindes vor. Obwohl vereinzelte Parallelen im Umgang mit dem Kind vorliegen, befasst sich NIDCAP tiefgehender mit neurologischer Entwicklungsförderung [23, 24].

\section{Gesundheitssystem}

Die Einführung des NIDCAP-Konzepts führt zu einer geringeren Aufenthaltsdauer, zu weniger durch Unreife bedingten Erkrankungen und somit zu niedrigeren Behandlungskosten. Deshalb stellt das Gesundheitssystem in Deutschland kein Problem für die Umsetzung des Konzepts dar.
Die NIDCAP-Ausbildung ist anspruchsvoll, aber nicht unmöglich. Die EFIB-Qualifizierung kann die Implementierung des NIDCAPKonzepts erleichtern, ersetzt sie aber nicht.

Was in anderen Ländern schon lange funktioniert, kann und muss auch in Deutschland möglich gemacht werden: Zum einen wegen der immer besseren Überlebenschancen auch sehr kleiner Frühgeborener und den daraus resultierenden steigenden Behandlungskosten, die mittels NIDCAP erheblich gesenkt werden können. Und wegen des besseren Erwerbs neurologischer und sozialer Kompetenzen durch gezielte Förderung der Hirnentwicklung, Stärkung der Familienbindung und daraus folgender geringerer Traumaschäden bei Eltern und Kindern.

\section{Literatur}

1 VandenBerg KA. Basic competencies to begin developmental care in the intensive care nursery. In: Infants and Young Children 1993; 6:52-59

2 Schothorst PF. Developmental Impact of Neonatal Stress. A Neuropsychiatric Study of Neonatal Intensive Care Unit Graduates at Schoolage, 1990

3 VandenBerg KA. Individualized Developmental Care for High Risk Newborns in the NICU, a Practice Guideline. In: Early Human Development 2007; 83:433-442

4 Altimier LB, Eichel M, Warner B, Tedeschi L, Brown B (2004): Developmental Care, Changing the NICU Physically and Behaviorally to promote Patient Outcomes and Contain Costs. In: Neonatal Intensive Care 2007; 17(2):35-39

5 Als H, Gilkerson L, Duffy FH, McAnulty GB, Buehler DM, VandenBerg K, Sweet $N$, Sell E, Parad RB, Ringer SA, Butler SC, Blickman JG, Jones KJ. Three-Center, randomized, Controlled Trial of Individualized Developmental care for VLBW Preterm Infants: Medical, Neurodevelopmental, Parenting and Caregivin Effects. In: Developmental and Behavioral Pediatrics 2003; 24(6):399-408

6 Kenner C, McGrath JM. Developmental Care of Newborn and Infant. A Guide For Health Professionals. USA: Elsevier; 2004

7 Universitätsklinikum Düsseldorf. Online im Internet: http://www.uniduesseldorf.de/Intensivpflege/seiten_ft/pdf/verveur.pdf; Zugriff: 12.05.2010

8 NIDCAP Federation International. Online im Internet: http://www.nidcap. org; Zugriff: 06.10.2010

9 Als H. Program Guide. Newborn individualized care and assement Program (NID$(A P)$ - an education and training program for health care professionals. Online im Internet: http://www.nidcap.org/file.aspx?fileid=pg ; Zugriff: 02.10 .2010

Weitere Literatur online unter Thieme Connect.

\section{AUTORIN}

Stephanie Rist

ist examinierte Kinderkrankenschwester und arbeitet auf der Intensivstation der DRK-Kinderklinik Siegen. Wellersbergstraße 60, 57072 Siegen E-Mail: stephierist@web.de

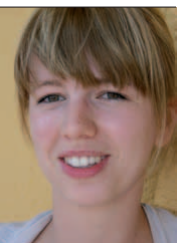

DOI 10.1055/s-0031-1281475

Intensiv 2011; 19 (5): 254-258

(c) Georg Thieme Verlag KG

Stuttgart . New York . ISSN 0942-6035 